\title{
Belphégor
}

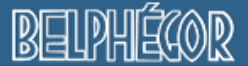

Littérature populaire et culture médiatique

13-1 | 2015

Distinctions That Matter/Fictions Économiques

\section{Xavier Fournier, Super-héros: une histoire française}

\section{Chris Reyns-Chikuma}

\section{(2) OpenEdition}

\section{Journals}

Édition électronique

URL : http://journals.openedition.org/belphegor/566

DOI : 10.4000/belphegor.566

ISSN : 1499-7185

Éditeur

LPCM

\section{Référence électronique}

Chris Reyns-Chikuma, "Xavier Fournier, Super-héros: une histoire française », Belphégor [En ligne], 13-1 | 2015, mis en ligne le 09 mai 2015, consulté le 22 septembre 2020. URL : http://

journals.openedition.org/belphegor/566; DOI : https://doi.org/10.4000/belphegor.566

Ce document a été généré automatiquement le 22 septembre 2020.

\section{(c) (i) (9)}

Belphégor est mis à disposition selon les termes de la Licence Creative Commons Attribution - Pas d'Utilisation Commerciale - Pas de Modification 4.0 International. 


\section{Xavier Fournier, Super-héros: une histoire française}

Chris Reyns-Chikuma

\section{RÉFÉRENCE}

Xavier Fournier, Super-héros: une histoire française, Huginn \& Muninn, 2014, 238 p.

ISBN-13: 978-2364801271 
1 Ceci pourrait être un «livre de table à café » [coffee table book]; et il l'est un peu par ses qualités matérielles, son grand format, sa typographie claire, la qualité de ses nombreuses images, en noir et blanc et en couleurs, petites et grandes (pleine page); mais il est aussi beaucoup plus. Accompagné d'un texte bien écrit et très instructif, tout fan, amateur ou spécialiste, en tirera beaucoup d'informations et de plaisir.

De par son titre, juxtaposant le superhéros, "nécessairement " américain, et une histoire française, il aurait pu ranimer les débats, parfois amers, entre les États-Unis et la France, qui ont eu lieu

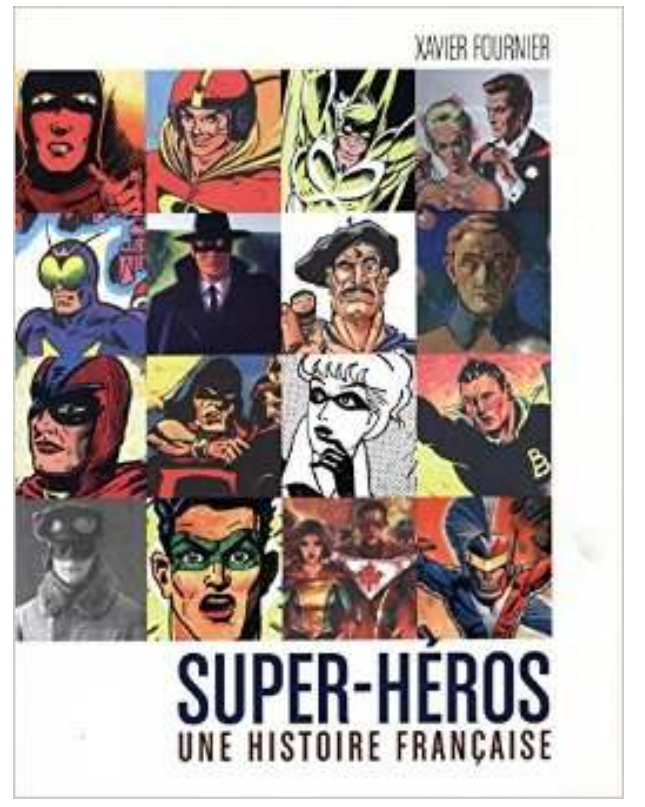
lors du centenaire de la naissance de la bande dessinée (voir «A Disputed Centenary " par Ann Miller). Cette fois, la question ne porte pas sur le média même mais sur un de ses genres les plus importants en termes commerciaux et symboliques, le genre "super-héros" qui est connu dans le monde entier pour être américain. Fournier ne conteste pas ce fait mais s'attache à montrer sérieusement que l'origine du genre a de multiples racines situées un peu partout dans le monde, aux États-Unis évidemment, mais aussi en Italie, en Espagne et en France.

3 Rappelant brièvement au lecteur que la définition du super-héros peut varier en fonction des critères utilisés (pouvoir surhumain, masque, double identité, ...), l'auteur montre que des racines profondes ancrent le super-héros dans la France du XIX siècle. Ceci est particulièrement crédible si l'on considère le rôle que le masque et la double identité ont joué entre autres à cause des changements rapides et soudains de régimes, huit en cent ans, connus par le pays. Ceux-ci impliquaient en effet pour certains individus «trop » engagés un changement d'identité et/ou un déguisement (faux nom, etc.) pour survivre dans le nouveau système politique. Ainsi s'explique, au moins partiellement, le succès d'un Monte-Cristo et des nombreux romans populaires du XIX siècle. Procédant chronologiquement des premières années du XIX siècle jusqu'à l'époque contemporaine, l'auteur se permet aussi dans chaque chapitre quelques sauts temporels. Il indique par exemple que Batman peut être considéré comme le descendant de Monte-Cristo (p.17, citant Umberto Eco) vu les nombreuses ressemblances (vengeance, double identité....). Une autre source probable de Batman est sans doute le Nemo des Vingt mille lieues sous les mers, surtout si l'on fait le parallèle entre l'arsenal héroïque privé de Nemo et la bat-cave (p.52). Malgré cette prépondérance et richesse des sources françaises, il ne s'agit pourtant nullement de dire qu'elles sont les seules qui peuvent expliquer les super-héros, car comme le dit joliment l'auteur, " on a tous deux parents, quatre grands parents, ... » (p. 18), et aussi d'ailleurs plus d'un seul descendant. L'influence se renverse avec un super-héros français comme Fantax, publié juste après la Libération et déjà marqué par les superhéros américains, à la fois sur un plan général (personnage, intrigue, etc.) et aussi stylistiquement puisque les auteurs de Fantax décalquaient les cases de Tarzan (p. 132). 
C'était une pratique courante dans le métier à l'époque mais qui va devenir de moins en moins commune avec le développement des droits internationaux.

L'auteur montre par ailleurs dans d'autres chapitres comment plusieurs personnages se sont développés hors du contrôle de leurs créateurs, comme Fantômas en Italie, puis au Mexique, sur des décennies et dans des centaines d'épisodes où Fantômas, devenu bon, rencontre Gandhi, Picasso, Hitler, puis le fils d'Hitler, des dictateurs sud-américains, l'actrice Claudia Cardinale, et bien d'autres personnages encore.

5 Presque aucune des "copies» françaises de super-héros ne vivra plus de quelques années. Alors pourquoi ne se sont-ils pas développés jusqu'à être aussi intéressants que les super-héros américains ? L'auteur ne donne pas de réponse claire. Fournier montre bien que ces super-héros français étaient le plus souvent de pâles imitations à la fois des Américains et des proto-super-héros français comme Monte-Cristo. On apprend aussi comment la Loi du 18 juillet 1949 (p. 147-48) influença le développement de la bande dessinée en décourageant la création de super-héros français. L'influence de cette Loi n'est pas à sous-estimer, puisqu'elle mènera au moins à une condamnation à la prison ferme pour le dessinateur Mouchot (p.169). L'une des formes de censure en dehors des plus connues (violence, érotisme) est une certaine masquophobie, c'est-àdire le rejet de l'usage du masque pour les héros (p.149). Une autre s'est faite au nom du rationalisme laïque, par la phobie de la force innée du super-homme, qui orientera le développement du genre super-héros vers diverses formes de science fiction, exploratoires et didactiques, où chaque phénomène extraordinaire devra être au moins explicable, si ce n'est clairement expliqué (p. 150). Enfin Fournier nous montre que, pour éviter le regard scrutateur de la censure, les histoires de super-héros se verront publiées dans des endroits inattendus, comme ce fut le cas du super-héros Fulguros publié dans Sylvie, magazine pour petites filles, en 1954 (p. 153). Enfin un autre critère pouvant expliquer le faible développement des super-héros en France est suggéré par plusieurs auteurs de bande dessinée, comme Serge Lehman, qui affirme: «je pense qu'il y a vraiment un schisme qui intervient culturellement autour de la notion même de super-héros, qui est une réaction épidermique, culturelle et sociale à l'Ubermensch des Allemands » (p. 225). Le chapitre sur les parodies présente de nombreux exemples dont certains sont biens connus, comme SuperDupont (p. 173-76) mais d'autres beaucoup moins, comme le film Mister Freedom de 1969. Les deux derniers chapitres mettent l'accent d'une part sur la renaissance littéraire prometteuse (avec Les Partisans, La Brigade chimérique, Les Sentinelles, ...) et sur l'action sociale des « super-héros du réel ».

Dans cet essai, l'auteur mêle des informations et réflexions connues des spécialistes de littérature et des médias populaires français (beaucoup que l'on trouvera d'ailleurs dans la revue Belphégor sous forme d'articles, de numéros spéciaux, ou de références à des études ou conférences) comme celles portant sur Fantômas, le Fantôme de l'opéra, Tarzan ou Rocambole, avec des informations nombreuses et riches mais moins connues sur des héros oubliés aujourd'hui, que seul un historien amateur comme Founier pouvait connaître aussi bien. Rappelons parmi ces héros l'Oiselle, le Masque Rouge, Yordi, Démonax, Nyctalope, Fantax, Atomas, Superboy, Fantomette, et les personnages issus du magazine Strange. Les informations sont toujours présentées dans une perspective originale puisqu'il s'agit d'explorer de possibles connections avec le mythe du super-héros. Parfois ces connections sont évidentes ou montrées par l'auteur de manière convaincante; d'autres fois la démonstration est générale et présente des liens supposés, mais sans preuves empiriques; d'autres fois encore, c'est le contraire qui se 
produit. Les liens sont supposés évidents et l'auteur montre qu'ils sont ténus. Tous les chapitres ne se valent donc pas. Bien qu'intéressants pour les informations qu'ils offrent, certains chapitres semblent moins crédibles ou plus anecdotiques. Ainsi le deuxième chapitre, sur les femmes masquées, les Amazones, et la masquomanie est basé sur des événements très ponctuels en France, qui se sont sans doute déroulés aussi dans d'autres capitales européennes à peu près à la même époque. Plus convaincant est le chapitre sur les années de l'Occupation où sont montrées les «frontières poreuses entre fiction et réalité » lorsque des résistants comme les Boulanger ou les Vistel prennent le nom de super-héros comme Fantômas, ou quand un Del Duca qui en profite pour collaborer en surface et résister en cachette par des tracts (ici l'auteur cite sa source, Isabelle Antonutti). Il présente toutefois souvent d'intéressantes pistes que peut-être d'autres chercheurs pourront explorer.

7 Outre son caractère hautement informatif, ce livre présente de nombreuses qualités. Ainsi l'auteur fait preuve d'une certaine sensibilité au sexisme et colonialisme, voire au racisme des bandes dessinées de super-héros. Une autre originalité de cet essai, c'est de montrer les multiples connexions qui existent entre ces personnages issus de romans populaires et les super-héros américains. En outre, il parvient à mettre en évidence la dynamique de circulation entre les divers genres et médias dès le $\mathrm{xIX}^{\mathrm{e}}$ siècle (journalisme, théâtre, romans, feuilleton, bande dessinée).

Si rien n'est dit de l'identité de Fournier qui, comme «ses» super-héros, privilégie l'anonymat, on se souviendra peut-être qu'il est l'auteur de deux autres ouvrages (Looney Tunes et Catch Heroes) et qu'il est l'un des rédacteurs de "Comic Box », magazine spécialisé sur les comics américains.

On regrettera peut-être que certaines questions ne soient pas abordées, par exemple le fait que Paris n'est pas le centre de ces créations de super-héros français. C'est en effet à Lyon et à Tourcoing qu'ils prospèrent. Ceci renforcera peut-être la théorie de la marginalisation géographique des paralittératures. On regrettera également un déséquilibre dans la bibliographie. Alors que des sources peu connues sont citées comme Bernard Trout pour une période complexe comme celle de l'Occupation, d'autres sources évidentes ne sont pas présentées (comme Pascal Ory, historien de la culture, y compris de la bande dessinée, et de l'Occupation). Le livre contient aussi parfois des coquilles bizarres dans les noms ou dates (2011 au lieu de 1911, p. 84). Il se termine par un bref index et une bibliographie, très courte et surtout arbitraire et désordonnée (pas d'ordre, ni alphabétique, ni chronologique). En dépit de ces défauts mineurs, cela reste pourtant un livre que toute bibliothèque et tout amateur devrait posséder, d'autant que son prix est raisonnable pour sa qualité (40 euros/63cad). 